This journal is the official publication of Bangladesh Society of Physiologists (BSP)

Web URL: www.banglajol.info/index.php/JBSP

Abstracted /indexed in Index Copernicus, Director of Open Access Journal, HINARI Index Medicus for South East Asia Region, Google Scholar, 12OR, infobse index, Open J gate, Cite factor, Scientific indexing services

pISSN-1983-1213; e-ISSN-2219-7508

\title{
Article
}

Article information:

Received: $15^{\text {th }}$ October 2020

Accepted: $7^{\text {th }}$ December 2020

DOI: https://doi.org/10.3329/jbsp.v15i2.50922

Corresponding author:

Sultana Ferdousi, Department ofPhysiology, Bangabandhu Sheikh Mujib Medical University, Dhaka. E-mail: sferousiratna@bsmmu.edu.bd sferdousiratna@gmail.com

Cite this article:

Rejwana I, Rimi SA, Sultana S, Ferdousi S. Cardiovascular responses to tilting in Type 2 Diabetic patients.

J Bngladesh Soc Physiol 2020;15(2): 78-84

This article is open access licensed under CC BY NC SA which allows readers copy, distribute, display, and perform the work and make derivative works based on it only for noncommercial purposes.

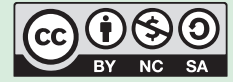

\section{Cardiovascular responses to tilting in Type 2 Diabetic patients}

\author{
Iffat Rejwana ${ }^{1}$, Sadia Afrin Rimi ${ }^{2}$,Shamima Sultana ${ }^{1}$, \\ Sultana Ferdousi ${ }^{1}$ \\ 1. Department of Physiology, Bangabandhu Sheikh Mujib Medical \\ University,Dhaka \\ 2. Department of Physiology, Ragib Rabeya Medical College, Sylhet.
}

\section{Abstract}

Background: Diabetes mellitus (DM) is a disorder with a debilitating effects on cardiac autonomic control. Multiple major cardiovascular risk factors associated with DM led diabetic patients at high risk of Cardiovascular Disease. Objective: To assess cardiovascular responses to tilting in Type 2 Diabetic patients (T2DM) with normal and abnormal autonomic function test. Methods: This experimental study was conducted on 60 patients of T2DM. Among them, 30 patients were with normal cardiovascular reflex test (group DN) and 30 patients were with abnormal test (group DA). Thirty(30) apparently healthy subjects with similar age and sex without any physical illness were enrolled as control. Tilt table test of all subjects was done by tilting at $60^{\circ}$ for $10 \mathrm{~min}$ by using a motorized tilt table. Cardiovascular response to tilt test was assessed by calculating $\Delta$ Heart rate (Acceleration index and Brake index); $\triangle \mathrm{SBP}\left(\mathrm{SBP}_{30 \mathrm{~s}-0}\right.$ and $\left.\mathrm{SBP}_{1 \text { min }-0}\right), \Delta \mathrm{DBP}$ $\left(\mathrm{DBP}_{30 \mathrm{~s}-0}\right.$ and $\left.\mathrm{DBP}_{1 \text { min }-0}\right)$ after tilting. For statistical analysis, one-way ANOVA followed by Bonferroni post hoc was used. Results: In this study, the Acceleration index was significantly higher in patient group DN compared to control and $\mathrm{DA}(\mathrm{p}<0.001)$. But the Brake index was significantly $(\mathrm{p}<0.01, \mathrm{p}<0.05)$ lower in both group of patients compared to control. In addition, $\mathrm{SBP}_{30 \text { sec- } 0}$ and $\mathrm{SBP}_{1 \text { min- } 0}$ were significantly higher in DA than those of control and DN.DBP ${ }_{30 \text { sec-0 }}$ and $\mathrm{DBP}_{1 \text { min-0 }}$ were significantly $(\mathrm{p}<0.001)$ lower in DA patients compared to DN and control. Conclusion: This study concluded that cardiovascular response to tilting was weak in T2DM patients and it was greatly affected in T2DM patients with abnormal autonomic function test.

Keywords: Cardiovascular response, Tilt table test, CAFT, Acceleration index, Brake index, $\mathrm{SBP}_{30 \text { sec- } 0}, \mathrm{SBP}_{1 \text { min- } 0} \mathrm{DBP}$ ${ }_{30 \text { sec- } 0}$ DBP $_{1 \text { min- } 0}$, Diabetic subjects. 
Introduction

D iabetic autonomic neuropathy (DAN) is the commonest complication which involves autonomic nervous system in patients with DM and one of its subtype is cardiovascular autonomic neuropathy (CAN). ${ }^{1}$ Clinical appearance of CAN may be preceded by a prolonged sub-clinical stage. ${ }^{1-2}$ Clinical CAN or late stage CAN occurs due to both parasympathetic and sympathetic denervation of heart. ${ }^{1}$ In addition, clinical CAN presents abnormal findings in noninvasive conventional autonomic function tests (CAFTs) which have been used to assess the function of autonomic nervous system(ANS). ${ }^{2}$ On the other hand, subclinical CAN or early stage of CAN is characterized by predominant damage to vagus nerve innervating the heart with subsequent compensatory increase in sympathetic drive of the heart resulting into resting cardiac autonomic imbalance characterized by resting tachycardia. ${ }^{1-}$

${ }^{2}$ Similar other studies also reported the presence of parasympathetic dysfunction at early stage of DM. ${ }^{3}$ However, subclinical CAN ensues largely from functional alterations of autonomic nerves and is considered to be a reversible disorder. ${ }^{4}$ By assessing the changes in heart rate and blood pressure in response to such stimuli one can assess the status of ANS outflow. ${ }^{5}$ In 1978, Ewing and Clarke introduced a sequence of five cardiovascular reflex tests known as classical or CAFT. These safe and noninvasive tests can efficiently assess the integrity of the baroreceptor reflex. ${ }^{6-8}$

Passive orthostatic test by a tilt table is a better choice to find the cardiovascular changes induced by upright postural change than active standing. ${ }^{9}$ A passive tilt test is a simple test and it can measure changes in both heart rate and blood pressure due to orthostatic stress. ${ }^{10}$ The effect of gravitational stress may cause shifting of central blood volume to dependent lower limb causing drop of BP. This is counter balanced by activating cardiovascular autonomic regulation to minimize the drop of BP. This adjustment is accompli-shed by transient rise of heart rate and vasoconstriction. ${ }^{11-12}$

HR increases immediately after tilting followed by mild bradycardia and then increase to steady values in healthy person. ${ }^{9}$ This rate of initial increment of HR after tilting can be expressed in a mathematical deduction-Acceleration index. In addition, the mild decrement of HR after the phase of initial tachycardia in angulated tilt state can be expressed by Brake index. In Diabetic patients with intact autonomic reflex, immediate rise of HR after tilting was significantly higher and the follow up decreased HR was slightly less compared to the healthy individual. On the other hand in Diabetic with abnormal autonomic function the HR increase was similar to control values but rebound bradycardia was less. So the acceleration index was higher in diabetic patients without neuropathy than the healthy person and in diabetic patients with neuropathy it is lowest. Whereas in case of brake index, it is much higher in healthy subjects than both group of diabetic patients with or without neuropathy. ${ }^{9}$ The systolic BP (SBP) declined abruptly after tilting up then returned to baseline values by 20 seconds.Initial change of the Diastolic Blood Pressure(DBP) is similar to SBP. Just after tilting up it also drop from the resting supine value.Then it rises to maximum value within first 30 second after tilting.In previous studies they found no significant difference between values obtained at first minute and those at second and third minute of tilting in healthy subjects.In diabetic patients without neuropathy, the observed pattern of DBP change with time after tilting was almost similar to that of healthy subjects. But in diabetic patients with neuropathy, though initially the DBP declined but within 20 second it reached to pre tilt supine level. ${ }^{9-10}$ Very few reports are published on the tilt response in Type 2 diabetic patients.Further report on this characteristic tilt response in case of T2DM with autonomic neuropathy is very scarce. To explore the nature

Volume 15 No. 2 December 2020: 78-84 
and extent ofcardiovascular autonomic regulation in type 2 diabetics with functional autonomic neuropathy subjected to gravitation stress, this study has been designed to observe the difference in cardiovascular response to orthostatic stress in type 2 Diabetic patients with or without abnormal autonomic function test by comparing tilt response between diabetics and healthy control as well as between T2DM patients with or without abnormal autonomic function test.

Methods

This experimental study was carried out from March 2019 to August, 2020 at the Department of Physiology, Bangabandhu Sheikh Mujib Medical University (BSMMU), Shahbag, Dhaka. For this study, total 60 male T2DM patients and 30 apparently healthy male subjects without any physical illness were enrolled by purposive sampling.Diabetic patients were further divided into the 2 subgroups according to the outcome of CAFT. Thirty (30) patients with normal CAFT were designated as DN and thirty (30) patients with abnormal CAFT was designated as DA. For both group, age range of the patients were 45-65 years and BMI were $18.5 \mathrm{~kg} / \mathrm{m}^{2}-29.9 \mathrm{~kg} / \mathrm{m}^{2}$. Similar age sex and BMI healthy subjects were control. The aim and procedure of this study was explained and informed written consent was taken from the participants. Then a detailed personal, medical,familyand dietary history was taken. A thorough physical examination of the subjects was done. Height and weight were taken and BMI was calculated. Then for biochemical tests, $8 \mathrm{ml}$ of venous blood was collected under aseptic precautions and ECG (MAC400 3 channel ECG machine, General Electric Company, India) was also done. All the subjects were free from thyroid disorder, anemia, liver disease, renal and cardiovascular disease.

Conventional autonomic function tests (CAFT) These tests were performed as previously described. ${ }^{5}$ The HR was monitored by ECG directly and continuously (MAC 4003 channel ECG machine, General Electric Company, India).For assessing parasympathetic reactivity, HR variation during deep breathing, immediate HR response to standing and HR response to the Valsalva maneuver is observed. For sympathetic reactivity, $\mathrm{BP}$ response to standing and $\mathrm{BP}$ response to sustained isometric handgrip tests are done. By these tests, Type 2 diabetic patients with and without autonomic dysfunction were classified..$^{7-8}$ If 2 or more abnormal HR-based test are present the person was considered to have parasympathetic neuropathy, and to detect sympathetic neuropathy, at least one abnormal test results out of two BP-based test was present. ${ }^{7}$

\section{Tilt test}

Before head up tilt test, the subjects were instructed to fast for 1 hour and they were requested not to drink or smoke during that time.They were asked for emptying bladder just before the test and the room temperature was maintained at $25^{\circ} \mathrm{C}$. All the subjects were given rest for 10 minutes lying on the tilt table. Supine HR, SBP and DBP were recorded. Then tilting was done at $60^{\circ} \mathrm{C}$ for 10 minutes by a motorized tilt table (Hi-Lo Mode; 220 volt; Cat No: IEMR4093HL;International Elecro Medical Co, India).Data were recorded for every minute for 10 minutes after tilting. After 10 minutes the tilt table was brought back to the horizontal position. The subjects were asked to avoid the movement of the lower limb during the procedure and also instructed if he felt any dizziness, lightheadedness or discomfort then he must inform it to the operator, so that the tilting would be ceased immediately and the table would be brought back to the horizontal position. BP were recorded by an automated sphygmomanometer (HME-7120, Omron Health Care Co. Ltd., Vietnam). As the subjects were in middle age,for their safety $\mathrm{SpO} 2$ was monitored throughout the procedure by a pulse oximeter (YK-88 LED, Yonker, China).

Cardiovascular response to tilt test was assessed by calculating $\Delta$ Heart rate (Acceleration index 
and Brake index); $\triangle \mathrm{SBP}\left(\mathrm{SBP}_{30 \mathrm{~s}-0}\right.$ and $\mathrm{SBP}_{1}$ min -0$) \triangle \mathrm{DBP}\left(\mathrm{DBP}_{30 \mathrm{~s}-0}\right.$ and $\left.\mathrm{DBP}_{1 \text { min }-0}\right)$ after tilting. ${ }^{10}$

From the ECG tracing,Acceleration index and Brake index were calculated as previously described. ${ }^{10}$ Acceleration index represents the maximum rise in $\mathrm{HR}$ response immediately after tilt by the percent of rise from the pre-tilt HR. Similarly, Brake index represents the return of HR towards pre-tilt by calculating the percentage of change from the maximum response due to tilt. ${ }^{9-10}$

SBP change after tilting was quantified by calculating $\mathrm{SBP}_{30 \text { sec-0 }}, \mathrm{SBP}_{1 \text { min-0 }}$ in one earlier study. ${ }^{9} \mathrm{SBP}_{30 \mathrm{sec}-0}$ represents the difference between maximum fall of SBP in 30 seconds after tilt and the value of the pre-tilt SBP. Similarly $\mathrm{SBP}_{1 \text { min-0 }}$ represents the difference between the value(fall) of SBP in 1 minute after tilt and the value of the pre-tilt SBP. These two values of SBP change indicate the extent of fall of SBP at two different time points after tilting.

DBP response to passive orthostatic stress was indicated by the rise of DBP with time after tilting which was observed in all groups. DBP changes with time after tilt indicated the extent of rise of DBP at two different time points after tilting. ${ }^{9}$

\section{Statistical analusis:}

Data were expressed as Mean \pm SD. Statistical analysis was done using SPSS version 22. Oneway ANOVA followed by Bonferroni post hoc, was done. $p$ value of $<0.05$ was considered as statistically significant.

\section{Results}

All subjects were similar by age and BMI. In this study, resting pulse rate, resting SBP and DBP) before tilt was similar in all three groups (Table I).

\section{Acceleration index and Brake index}

After application of passive orthostatic stress by tilting maneuvers, the acceleration index was found significantly higher in group $\mathrm{DN}(\mathrm{p} \leq 0.001)$ compared to control but it was significantly lower in DA compared to $\mathrm{DN}(\mathrm{p} \leq 0.001)$ and control $(\mathrm{p} \leq 0.05)$ (Table II). On the other hand, the Brake index was significantly lower in $\mathrm{DN}(\mathrm{p} \leq 0.001)$ and DA $(p \leq 0.001)$ than those of control. Furthermore, no difference was found in this parameter between group $\mathrm{DN}$ and $\mathrm{DA}$. (Table II)

Table I : Characteristics of the three groups of subjects included in the study $(\mathrm{N}=90)$

\begin{tabular}{lccc}
\hline Groups & $\mathrm{DN}(\mathrm{n}=30)$ & $\mathrm{DA}(\mathrm{n}=30)$ & Control $(\mathrm{n}=30)$ \\
\hline Age $($ Years $)$ & $52.57 \pm 4.92$ & $55.60 \pm 5.69$ & $52.90 \pm 5.39$ \\
BMI $\left(\mathrm{Kg} / \mathrm{m}^{2}\right)$ & $25.18 \pm 2.44$ & $25.4 \pm 2.44$ & $25.18 \pm 2.46$ \\
HR (beats/min) & $75.46 \pm 11.89$ & $72.96 \pm 8.24$ & $76.83 \pm 7.17$ \\
SBP (mm Hg) & $123.73 \pm 11.08$ & $126.83 \pm 12.11$ & $122.33 \pm 8.69$ \\
DBP $(\mathrm{mm} \mathrm{Hg})$ & $76.13 \pm 7.22$ & $78.50 \pm 7.65$ & $77.33 \pm 6.62$ \\
\hline
\end{tabular}

Data were expressed as mean \pm SD. Statistical analysis were done one way ANOVA followed by Bonferroni; DN-Diabetic patients with normal CAFT.DA-Diabetic patients withabnormal CAFT. $n$ - Number of the subjects in each groups; $\mathrm{N}$ - total number of subjects.HR-heart rate. SBP-Systolic blood pressure.DBP- Diastolic blood pressure. 
Table II: Indexes used to evaluate the cardiovascular responses induced by tilting up(N=90)

\begin{tabular}{lccc}
\hline Variables & $\mathrm{DN}(\mathrm{n}=30)$ & $\mathrm{DA}(\mathrm{n}=30)$ & Control(n=30) \\
\hline Acceleration index (\%) & $14.48 \pm 3.03^{* * *}$ & $10.01 \pm 2.42^{*} \cdots$ & $11.97 \pm 2.55$ \\
Brake index (\%) & $3.25 \pm 1.14^{* * *}$ & $3.39 \pm 1.50^{* * *}$ & $4.95 \pm 1.75$ \\
$\mathrm{SBP}_{30 \mathrm{sec}-0}(\mathrm{~mm} \mathrm{Hg})$ & $-6.10 \pm 4.91^{* *}$ & $-13.73 \pm 5.03^{* * *} \ldots$ & $-2.83 \pm 1.28$ \\
$\mathrm{SBP}_{1 \text { min-0}}(\mathrm{mm} \mathrm{Hg})$ & $-2.43 \pm 5.17$ & $-14.86 \pm 4.05^{* * *} \ldots$ & $-2.46 \pm 3.98$ \\
DBP $_{30 \text { sec-0 }}(\mathrm{mm} \mathrm{Hg})$ & $3.20 \pm 4.63$ & $-0.50 \pm 4.97^{*}$. & $5.23 \pm 1.67$ \\
DBP $_{1 \text { min-0 }}(\mathrm{mm} \mathrm{Hg})$ & $4.93 \pm 4.19$ & $-0.30 \pm 3.06^{* *} \ldots$ & $5.53 \pm 2.28$ \\
\hline
\end{tabular}

Data were expressed as mean $\pm \mathrm{SD}$. Statistical analysis were done by one way ANOVA followed by Bonferroni; SBP ${ }_{30 \mathrm{~s}-0}$-Baseline and 30 seconds tilt difference, $\mathrm{SBP}_{1 \mathrm{~min}-0}$-Baseline and 1 minute tiltdifference; $\mathrm{DBP}_{30 \mathrm{sec}-0}$-Baseline and 30 seconds tilt difference, $\mathrm{DBP}{ }_{1 \mathrm{~min}-0}$-Baseline and 1 minute tilt difference. Group DN- Diabetic patients with normal CAFT; Group DA- Diabetic patients with abnormal CAFT; Control-apparently healthy subjects; $N=$ total number of subjects; ${ }^{*} \mathrm{p} \leq 0.05$ vs. control; $\mathrm{p} \leq 0.05$ vs. group $\mathrm{DN}^{* *}$ : $\mathrm{p} \leq 0.01$ vs. . control; $\cdots: \mathrm{p} \leq 0.01$ vs. group $\mathrm{DN}^{* * *}$ : $\mathrm{p} \leq 0.001$ vs. control ; $\cdots: \mathrm{p} \leq 0.001$ vs. group DN.

\section{$\triangle S B P$ and $\triangle D B P$}

The result of SBP change with time in this study suggest maximum fall of SBP within 30 seconds of tilting but after these it tends to rise with time but still it was below the pre-tilt value at 1 minute after tilting.

In our study, the fall of SBP was slight and rise of DBP was moderate in control subjects at 30 second and at the first minute after tilting. In addition, significantly greater drop of SBP and lesser rise of DBP both at 30 seconds and at 1 minute after tilting was found in DA group compared to control and DN. Again the fall of $\mathrm{SBP}_{30 \text { sec- } 0}$ was significantly higher in DN than that of control. (Table II)

\section{Discussion}

The present study investigated the cardiovascular responses to orthostatic stress in Type 2 Diabetic patients by employing passive tilt table test. This study evaluated the difference in cardiovascular reaction to head up tilt (passive orthostatic stress) between the diabetic patients with normal and the abnormal autonomic function, as well as between healthy subjects and diabetic patients.

Volume 15 No. 2 December 2020: 78-84
In this study significantly higher acceleration index and lower brake index was found in diabetic patients with dysautonomia or normal autonomic function than control.Similar result on acceleration and brake index were reported by several studies. ${ }^{10}$ Navarro et al. ${ }^{9}$ observed similar change of Acceleration index but it was in Type 1 diabetic patients. They found higher Acceleration index in Type 1diabetic patients with normal autonomic function and reduced Acceleration index in diabetic patients with abnormal autonomic function compared to control. Our study also found similar pattern of acceleration index in type 2 diabetes compared to healthy control. Furthermore, it was even lower in patients with autonomic dysfunction than diabetes with normal autonomic function.

Similar to our study they also used cardiorespiratory reflex test to differentiate the diabetic patients with normal and abnormal autonomic function. But one similar previous study studied Acceleration index in the insulin dependent diabetic patients and compared data between short and long duration and between with or without retinopathy complication ${ }^{10}$. Though Navarro et al. ${ }^{9}$ did not find significant 
difference in Acceleration index, but they observed similar change of Brake index in DA group. The value of Acceleration index and Brake index suggestting the HR changes observed in our healthy control are close to the observation in their healthy subjects in Navarro et $\mathrm{al}^{9}$. But it was comparatively lower than those of Sundkvistet al. ${ }^{10}$ On the other hand Sundkvist, Lilja and Almer found significantly lower Brake index in diabetic patients with retinopathy. Moreover, they also found lower Brake index in diabetic patient with abnormal autonomic function and retinopathy. ${ }^{10}$

In our study, the SBP response to gravitational stress demonstrated drop of SBP within 30 seconds after tilting which was observed in all groups. But Sundkvistet al. ${ }^{10}$ used mean SBP and DBP values recorded after 1 minute and subsequently 10 minutes after tilting. The result of DBP change with time in this study suggest rise of DBP within 30 seconds and 1 minute of tilting. But after that it tends to fall with time, still it was above the pre-tilt value at 1 minute after tilting. These two values of DBP change indicate the extent of rise of DBP at two different time points after tilting. In addition,the expected changes in SBP and DBP at specified time period after tilt was greatly depressed in diabetics with autonomic dysfunction and less severely in diabetics with normal autonomic function in our study.

Navarro et al. began to observe the change in SBP and DBP from 5 seconds after tilt and they continued to monitor these changes up to 2 minutes. ${ }^{9}$ They found fall of SBP and DBP from 5 seconds. SBP fall was continued upto 20 seconds, then it started to rise. In case of DBP, after initial fall its value was increased to maximum by about 30 seconds. Thereafter it returned to pre-tilt level at 1 minute 9 . In our study, we recorded data earliest at 30 seconds after tilt. Therefore, we missed the initial fall of DBP.

The outcome of our study suggested greatly depressed tilt response in Diabetic patients with abnormal autonomic function test. It indicated severe error in compensatory circulatory adjustment to the postural challenge in this group of Diabetic patients with autonomic dysfunction which otherwise would occur by appropriate autonomic modulation. In addition, Diabetic patients without autonomic dysfunction in this study also demonstrated relatively weak autonomic modulation to the postural challenge.910,13-15

In our study, relatively greater Acceleration index and smaller Brake index in diabetic patients with normal autonomic function may be attributed to mild enhancement in parasympathetic withdrawn. The result of this greater Acceleration index and lower Brake index as well as greater drop of SBP and lesser rise of DBP immediately after tilt and lesser recovery with time in diabetic patients suggest enhanced parasympathetic inhibition and poor sympathetic compensatory activity in them which may be attributed to early stage damage in parasympathetic nervous system and damagein sympathetic nervous system occur inlate stage. ${ }^{3,5,9-10,16-17}$ Again this phenomenon was paradoxical in DBP change in Diabetic patients with abnormal autonomic function test. They showed severely depressed cardiovascular autonomic response to tilting by markedly abnormal heart rate and BP change immediately after tilting and little or almost no recovery with time.Previous studies also showed markedly reduced response to tilt in this group of patients compared to control. It suggested greatly reduced autonomic response to gravitational stress in Diabetic patients with autonomic dysfunction. In addition, reduced nerve conduction due to oxidative damage and nitrosative stress, decline in neuro-protective agents, synaptic degradation of organelles, extracellular matrix, etc. may be involved in developing autonomic neuropathy in diabetic patients. ${ }^{1,2,14,18}$

\section{Conclusion}

Based on the results of the study it can be concluded that cardiovascular response to

Volume 15 No. 2 December 2020: 78-84 
orthostatic stress induced by tilting was weak in Type 2 Diabetic patients and this weakness was more pronounced in Type 2Diabetic patientswith abnormal autonomic function.

Ethical consideration The protocol of this study was approved by the Institutional Review Board of BSMMU.

Conflict of interest Authors of this article declares no conflict of interest.

\section{References}

1. Kuehl M, Stevens MJ .Cardiovascular autonomic neuropathies as complications of diabetes mellitus. Nat Rev Endocrinol 2012; 8: 405-416. doi: 10.1038/ nrendo.2012.21.

2. Dimitropoulos G, Tahrani AA, Stevens MJ. Cardiac autonomic neuropathy in patients with diabetes mellitus. World J Diabetes2014; 5(1): 17-39

3. Ewing DJ, Clarke BF. Diagnosis and management of diabetic autonomic neuropathy. Brit Med J. 1982;285(6346): 916-18

4. Clemente de Souza Pereira Rolim L, de Sá JR, Chacra AR, Dib SA . Diabetic cardiovascular autonomic neuropathy: Risk factors, clinical impact and early diagnosis. Arq Bras Cardiol 2008; 90(4): 23-31.

5. Ewing DJ, Martyn CN, Young RJ, Clarke BF. The value of cardiovascular autonomic function tests: 10 years' experience in diabetes. Diabetes Care1985; 8: 49198.

6. Ewing DJ . Cardiovascular reflexes and autonomic neuropathy. Clin Sci Mol Med. 1978;55(4): 321-27.

7. Pathak A, Gupta S, Kumar S, Agarwal S. Evaluation of cardiovascular autonomic nervous functions in Diabetics: study in a rural teaching hospital. J Pract Cardiovasc Sci 2017; 3 (3): 150-57.

8. Fisher VL, Tahrani AA. Cardiac autonomic neuropathy in patients with diabetes mellitus: current perspectives. Diabetes, Metabolic Syndrome and Obesity: Targets and Therapy 2017;10:419-34. doi.org/10.2147/ DMSO.S129797

9. Navarro X, Kennedy W R and Ferrer M T . Cardiovascular responses to tilting in healthy and diabetic subjects. J Neurol Sci 1991; 104(1):39-45

10. Sundkvist G, Lilja B, Almer LO. Abnormal diastolic blood pressure and heart rate reactions to tilting in diabetes mellitus. Diabetologia 1980; 19(5):433-8.

11. Mukai S, Lipsitz LA. Orthostatic hypotension. Clin Geriatr Med 2002; 18: 253-68.

12. Lambert E, Lambert GW. Sympathetic dysfunction in vasovagal syncope and the postural orthostatic tachycardia syndrome. Front Physiol 2014; 5: 280.

13. Zhang Y, Critchley LA, Tam YH, Tomlinson B. Shortterm postural reflexes in diabetic patients with autonomic dysfunction. Diabetologia2004;47(2):30411.doi: $10.1007 / \mathrm{s} 00125-003-1286-2$.

14. Lutfi NM, Ameer AH . Electrodiagnostic evaluation of autonomic dysfunction in diabetes mellitus in Baghdad, Iraq. IOSR J Pharm 2018; 12(8): 27-32.

15. Philips JC, Marchand M andScheen AJ. Squatting, a posture test for studying cardiovascular autonomic neuropathy in diabetes. Diabetes Metab2011; 6(37):489-96.

16. Wieling W, Borst C, Brederode JFMV, Torman MAVD, Montfrans G AV, Dunning AJ . Testing for Autonomic Neuropathy: Heart rate changes after orthostatic aanoeuvres and static muscle contractions. Clin sci 1983; 64(6):581-6. DOI: 10.1042/cs0640581

17. Deepak A, Aithal K, Khode VH, Nallulwar SC. Short term heart rate variability for early assessment of autonomic neuropathy in patients with type 2 diabetes mellitus: A comparative cross-sectional study. Ann Nigerian Med 2014;8(1): 4-7.

18. Bandeira SM, Da Fonseca LJS, Da Silva Guedes G,Rabelo LA, Goulart MOF, Vasconcelos SML. Oxidative stress as an underlying contributor in the development of chronic complications in diabetes mellitus. Int J Mol Sci 2013; 14: 3265-84. Doi: 10.3390 /ijms 14023265. 